\title{
On weak minima of certain integral functionals
}

\author{
by Gioconda Moscariello (Salerno)
}

\begin{abstract}
We prove a regularity result for weak minima of integral functionals of the form $\int_{\Omega} F(x, D u) d x$ where $F(x, \xi)$ is a Carathéodory function which grows as $|\xi|^{p}$ with some $p>1$.
\end{abstract}

1. Introduction. This paper is concerned with the variational functionals of the form

$$
\mathcal{F}(u)=\int_{\Omega} F(x, D u) d x
$$

where $\Omega$ is an open subset of $\mathbb{R}^{n}, n \geq 2, u: \Omega \rightarrow \mathbb{R}^{m}, m \geq 1$, and $F: \Omega \times \mathbb{R}^{m n} \rightarrow \mathbb{R}$ is a Carathéodory function such that

$$
|\xi|^{p} \leq F(x, \xi) \leq \alpha|\xi|^{p}, \quad p>1 .
$$

The notion of the weak minimizer makes sense if $F$ satisfies the following Lipschitz type condition:

$$
|F(x, \xi)-F(x, \eta)| \leq \beta|\xi-\eta|\left(|\xi|^{p-1}+|\xi-\eta|^{p-1}\right)
$$

for some constant $\beta$.

Definition 1.1. A mapping $u \in W_{\mathrm{loc}}^{1, r}\left(\Omega, \mathbb{R}^{m}\right), \max \{1, p-1\} \leq r<p$, is called a weak minimizer of the integral (1.1) if

$$
\int_{\Omega}[F(x, D u+D \Phi)-F(x, D u)] d x \geq 0
$$

for all $\Phi \in W^{1, r /(r-p+1)}\left(\Omega, \mathbb{R}^{m}\right)$ with compact support.

If we assume that $F$ is differentiable with respect to the variable $\xi \in \mathbb{R}^{n m}$, we can write the Euler-Lagrange system for the functional (1.1). Then it

1991 Mathematics Subject Classification: 49J40, 49K20, 35B65.

Key words and phrases: weak minimizer, maximal functions.

This work was performed as a part of a National Research Project supported by M.U.R.S.T. 
turns out that any weak minimizer of (1.1) solves the equation

$$
\int_{\Omega} A(x, D u) D \Phi d x=0
$$

for all $\Phi \in W_{0}^{1, r /(r-p+1)}$, where $A(x, \xi)=D_{\xi} F(x, \xi)$. Note that $r /(r-p+1)$ $>p$ for $r<p$. For this reason we say that $u$ is a very weak solution of the Euler-Lagrange system.

The theory of very weak solutions of equations of type (1.4) has been initiated by T. Iwaniec and C. Sbordone. In [IS] they gave various results concerning existence and regularity of such solutions. Among other things they prove that if $r$ is close to $p$, then every $W_{\text {loc }}^{1, r}$-solution is in fact a $W_{\mathrm{loc}}^{1, p}$ solution. These results rely on new estimates for the Hodge decomposition which were introduced by $\mathrm{T}$. Iwaniec in $[\mathrm{Iw}]$. For related results see also [GLS], [Mo].

Later, J. Lewis [Le] offered another approach to the same problem using the theory of $A_{p}$-weights of Muckenhoupt.

In this paper we study the regularity of weak minimizers of integrals of type (1.1), under hypotheses (1.2) and (1.3). Special emphasis will be placed on the integrands $F(x, \xi)$ which are not necessarily differentiable. The main result is

THEOREM 1. There exists an exponent $r_{1}=r_{1}(m, n, p, \alpha, \beta)$ with $\max \{1, p-1\}<r_{1}<p$ such that if $u \in W_{\text {loc }}^{1, r}\left(\Omega, \mathbb{R}^{m}\right), r_{1} \leq r<p$, is a weak minimizer of the integral (1.1), then $u \in W_{\mathrm{loc}}^{1, p}\left(\Omega, \mathbb{R}^{m}\right)$.

In the case $r=p$ regularity results for minimizers of $\mathcal{F}(u)$ have been established in [GG], [Gi]. To prove Theorem 1 we follow the technique introduced by J. Lewis [Le]. Some results on the maximal functions and reverse Hölder inequalities will also be used.

2. Preliminaries. Let $B(x, r)=\left\{y \in \mathbb{R}^{n}:|y-x|<r\right\}$ and $|B(x, r)|$ denote its Lebesgue measure. For a measurable function $f$ on $\mathbb{R}^{n}$ we set

$$
f_{x, r}=f_{B(x, r)}|f(y)| d y=\frac{1}{|B(x, r)|} \int_{B(x, r)}|f(y)| d y .
$$

Denote the Hardy-Littlewood maximal function of $f$ by

$$
M f(x)=\sup _{r>0} f_{B(x, r)}|f(y)| d y
$$

and set

$$
M^{k} f(x)=M^{k-1}(M f)(x) \quad \text { for } k \geq 2 .
$$


Definition 2.1. For $1<p<\infty$, we say that a nonnegative measurable function $a \in L_{\text {loc }}^{1}\left(\mathbb{R}^{n}\right)$ is in the Muckenhoupt class $A_{p}$, or is an $A_{p}$-weight iff

$$
A_{p}(a)=\sup _{x \in \mathbb{R}^{n}, r>0}\left(f_{B(x, r)} a\right)\left(f_{B(x, r)} a^{-1 /(p-1)}\right)^{p-1}<\infty .
$$

The following lemma can be proved (see [Le] and [Do]).

LEMmA 2.2. Let $1<p<\infty$. There exists a positive constant $c=c(n, p)$ such that for any $0<2 \delta<p-1$, the function $(M f)^{-\delta}$ is an $A_{p}$-weight and $A_{p}\left((M f)^{-\delta}\right) \leq c$ for all $f \in L^{1}\left(\mathbb{R}^{n}\right), f \neq 0$.

Let us recall the fundamental result about $A_{p}$-weights due to Muckenhoupt (see $[\mathrm{Mu}])$.

Theorem 2.3. For $1<p<\infty$ and $a \in A_{p}$, there exists a positive constant $c=c\left(p, n, A_{p}(a)\right)$ such that

$$
\int_{\mathbb{R}^{n}} a(x)(M f(x))^{p} d x \leq c \int_{\mathbb{R}^{n}} a(x)|f(x)|^{p} d x
$$

for all $f \in L^{p}\left(\mathbb{R}^{n}, a\right)$.

If $a(x)=1$ a.e., then the previous result is just the Hardy-Littlewood maximal theorem.

We shall need several lemmas.

Lemma 2.4. Let $1<p<\infty, x_{0} \in \mathbb{R}^{n}, r>0$ and $B=B\left(x_{0}, r\right)$. If $f \in W^{1, p}(B)$ then there exists $c=c(n, p)$ such that for any $x \in B$,

$$
\left|f(x)-f_{x_{0}, r}\right| \leq \operatorname{crM}\left(|D f| \chi_{B}\right)(x)
$$

where $\chi_{B}$ is the characteristic function of $B$.

For the proof see Lemma 2.1 of [Le].

Lemma 2.5. Let $\lambda>0,1<q<\infty, x_{0} \in \mathbb{R}^{n}$ and $r>0$. Suppose $f \in W^{1, q}\left(\mathbb{R}^{n}\right)$, supp $f \subset B\left(x_{0}, r\right)$ and

$$
F(\lambda)=\{x: M(|D f|)(x) \leq \lambda\} \cap B\left(x_{0}, 2 r\right) \neq \emptyset .
$$

Then $f_{\mid F(\lambda)}$ has an extension to $\mathbb{R}^{n}$, denoted by $v=v(\cdot, \lambda)$, such that

(i) $v=f$ on $F(\lambda)$,

(ii) $\operatorname{supp} v \subset B\left(x_{0}, 2 r\right)$,

(iii) $v \in W^{1, \infty}\left(\mathbb{R}^{n}\right)$ with $\|v\|_{\infty} \leq c \lambda r$ and $\|D v\|_{\infty} \leq c \lambda$.

This is a slight modification of a lemma due to J. Lewis (see [Le] and also $[\mathrm{AF}]$ and $[\mathrm{Do}])$.

Finally, we shall need an amended form of a theorem of Gehring $[G]$ quoted in $[\mathrm{Gi}]$ and $[\mathrm{Gu}]$. 
Theorem 2.6. Let $R>0, q>1$ and $g \in L^{q}\left(B\left(x_{0}, R\right)\right)$ be such that

$$
f_{B(x, r / 8)}|g|^{q} d y \leq c\left(f_{B(x, r)}|g| d y\right)^{q}+\vartheta \underset{B(x, r)}{f}|g|^{q} d y
$$

for $0<\vartheta<1$ and $x \in B\left(x_{0}, R / 2\right), 0<r \leq R / 8$. Then there exist $c^{\prime}=$ $c^{\prime}(n, \vartheta, c, q)$ and $\eta=\eta(n, \vartheta, c, q)>0$ such that if $\tau=q(1+\eta)$ then

$$
\left(f_{B(x, R / 4)}|g|^{\tau} d y\right)^{1 / \tau} \leq c^{\prime}\left(f_{B(x, R / 2)}|g|^{q} d y\right)^{1 / q} .
$$

3. Proof of Theorem 1. Assume that $F: \Omega \times \mathbb{R}^{m n} \rightarrow \mathbb{R}$ is a Carathéodory function satisfying (1.2) and (1.3).

In the following we denote by $c$ a constant that depends only on $n, m$, $\alpha, \beta, p$ but may change from line to line.

Let $B=B\left(x_{0}, R\right) \subset \Omega$ for some $R \leq 1$. For fixed $y_{0} \in B\left(x_{0}, R / 2\right)$ and $0<\varrho<R / 8$, let $B_{\varrho}=B\left(y_{0}, \varrho\right)$ and $\varphi \in C_{0}^{\infty}\left(B_{2 \varrho}\right)$ such that $\varphi=1$ on $B_{\varrho}, 0 \leq \varphi \leq 1$ on $B_{2 \varrho}$ and $|D \varphi| \leq c \varrho^{-1}$. Set

$$
u_{4 \varrho}=f_{B_{4}} u(x) d x
$$

and $\widetilde{u}=\left(u-u_{4 \varrho}\right) \varphi, E(\lambda)=\left\{x \in \mathbb{R}^{n}: M(|D \widetilde{u}|) \leq \lambda\right\}$ and $F_{\lambda}=E_{\lambda} \cap B_{4 \varrho}$. Since supp $\widetilde{u} \subset B_{2 \varrho}$, for $x \in \mathbb{R}^{n}-B_{3 \varrho}$ we observe that

$$
M(|D \widetilde{u}|)(x) \leq c \varrho^{-n} \int_{B_{2 \varrho}}|D \widetilde{u}|(y) d y=\lambda_{0} .
$$

Therefore $F(\lambda)$ is not empty for $\lambda>\lambda_{0}$ and we may apply Lemma 2.5 with $f=\widetilde{u}$ and $r=2 \varrho$ to extend $\widetilde{u}_{\mid F(\lambda)}$ to $\mathbb{R}^{n}$. The extended function, denoted by $v$, will satisfy conditions (i)-(iii).

We use $v$ as a test function in Definition 1.1. Then from Lemma 2.5 and condition (1.2) we get

$$
\begin{aligned}
\int_{F(\lambda)}[F(x, D u)-F(x, D u & -D \widetilde{u})] d x \\
& \leq \int_{B_{4 \varrho}-F(\lambda)}[F(x, D u-D v)-F(x, D u)] d x \\
& \leq \beta \int_{B_{4 e}-F(\lambda)}|D v|\left(|D u|^{p-1}+|D v|^{p-1}\right) d x \\
& \left.\leq c \lambda \int_{B_{4 \varrho}-F(\lambda)}|D u|^{p-1}+c \int_{B_{4 \varrho}-F(\lambda)}|D v|^{p}\right) d x .
\end{aligned}
$$


We multiply both sides of this inequality by $\lambda^{-(1+\delta)}$ where $\delta=p-r$ will be chosen at the end of the proof, and integrate from $\lambda_{0}$ to $\infty$ :

$$
\begin{aligned}
& \int_{\lambda_{0}}^{\infty} \lambda^{-(1+\delta)} d \lambda \int_{B_{4}}[F(x, D u)-F(x, D u-D \widetilde{u})] \chi_{\{M(|D \widetilde{u}|) \leq \lambda\}} d x \\
& \leq c \int_{\lambda_{0}}^{\infty} \lambda^{-\delta} d \lambda \int_{B_{4 \varrho}-F(\lambda)}|D u|^{p-1} d x+\int_{\lambda_{0}}^{\infty} \lambda^{-(1+\delta)} d \lambda \int_{B_{4 \varrho}-F(\lambda)}|D v|^{p} d x .
\end{aligned}
$$

After interchanging the order of integration, the left hand side of (3.2) becomes

$$
\begin{aligned}
\int_{B_{4}-E\left(\lambda_{0}\right)} & {[F(x, D u)-F(x, D u-D \widetilde{u})] d x \int_{M(|D \widetilde{u}|)}^{\infty} \lambda^{-(1+\delta)} d \lambda } \\
& +\int_{\lambda_{0}}^{\infty} \lambda^{-(1+\delta)} d \lambda \int_{E\left(\lambda_{0}\right)}[F(x, D u)-F(x, D u-D \widetilde{u})] d x \\
= & \frac{1}{\delta} \int_{B_{4 \varrho}-E\left(\lambda_{0}\right)}[F(x, D u)-F(x, D u-D \widetilde{u})] M(|D \widetilde{u}|)^{-\delta} d x \\
& +\frac{\lambda_{0}^{-\delta}}{\delta} \int_{E\left(\lambda_{0}\right)}[F(x, D u)-F(x, D u-D \widetilde{u})] d x \\
\equiv & \frac{1}{\delta} J_{1}+\frac{\lambda_{0}^{-\delta}}{\delta} J_{2} .
\end{aligned}
$$

Since supp $\widetilde{u} \subset B_{2 \varrho}$ and $B_{4 \varrho}-E\left(\lambda_{0}\right)=B_{4 \varrho}-F\left(\lambda_{0}\right)$, we obtain

$$
\begin{aligned}
J_{1} & =\left(\int_{B_{4 \varrho}}-\int_{F\left(\lambda_{0}\right)}\right)[F(x, D u)-F(x, D u-D \widetilde{u})] M(|D \widetilde{u}|)^{-\delta} d x \\
& =\left(\int_{B_{2 \varrho}}-\int_{F\left(\lambda_{0}\right)}\right)[F(x, D u)-F(x, D u-D \widetilde{u})] M(|D \widetilde{u}|)^{-\delta} d x .
\end{aligned}
$$

Now, using the fact that $\widetilde{u}=u$ on $B_{\varrho}$ and $F(x, 0)=0$, from the previous relation we get

$$
\begin{aligned}
J_{1}= & \int_{B_{\varrho}} F(x, D u) M(|D \widetilde{u}|)^{-\delta} d x \\
& +\int_{B_{2 \varrho}-B_{\varrho}}[F(x, D u)-F(x, D u-D \widetilde{u})] M(|D \widetilde{u}|)^{-\delta} d x \\
& -\int_{F\left(\lambda_{0}\right)}[F(x, D u)-F(x, D u-D \widetilde{u})] M(|D \widetilde{u}|)^{-\delta} d x .
\end{aligned}
$$


Then from (3.2), (3.3) and (3.5),

$$
\begin{aligned}
\frac{1}{\delta} \int_{B_{\varrho}} F(x, D u) & M(|D \widetilde{u}|)^{-\delta} d x \\
\leq & \frac{1}{\delta} \int_{F\left(\lambda_{0}\right)}[F(x, D u)-F(x, D u-D \widetilde{u})] M(|D \widetilde{u}|)^{-\delta} d x \\
& +\frac{1}{\delta} \int_{B_{2 \varrho}-B_{\varrho}}[F(x, D u-D \widetilde{u})-F(x, D u)] M(|D \widetilde{u}|)^{-\delta} d x \\
& +\frac{\lambda_{0}^{-\delta}}{\delta} \int_{E\left(\lambda_{0}\right) \cap B_{2 \varrho}}[F(x, D u-D \widetilde{u})-F(x, D u)] d x \\
& +c \int_{\lambda_{0}}^{\infty} \lambda^{-\delta} d \lambda \int_{B_{4}-F(\lambda)}|D u|^{p-1} d x+c \int_{\lambda_{0}}^{\infty} \lambda^{p-1-\delta}\left|B_{4 \varrho}-F(\lambda)\right| d \lambda .
\end{aligned}
$$

Let us use (1.2) to estimate the left hand side from below, and the Lipschitz condition (1.3) to estimate the integrals on the right hand side. Since $\lambda_{0}^{-\delta} \leq M(|D \widetilde{u}|)^{-\delta}$ on $E\left(\lambda_{0}\right)$ we obtain

$$
\begin{aligned}
\int_{B_{\varrho}}|D u|^{p} M(|D \widetilde{u}|)^{-\delta} d x \leq & c \int_{E\left(\lambda_{0}\right) \cap B_{2 \varrho}}|D \widetilde{u}|\left(|D u|^{p-1}+|D \widetilde{u}|^{p-1}\right) M(|D \widetilde{u}|)^{-\delta} d x \\
& +c \int_{B_{2 \varrho}-B_{\varrho}}|D \widetilde{u}|\left(|D u|^{p-1}+|D \widetilde{u}|^{p-1}\right) M(|D \widetilde{u}|)^{-\delta} d x \\
& +c \delta \int_{\lambda_{0}}^{\infty} \lambda^{-\delta} d \lambda \int_{B_{4 \varrho}}|D u|^{p-1} \chi_{\{M(|D \widetilde{u}|)>\lambda\}} d x \\
& +c \delta \int_{\lambda_{0}}^{\infty} \lambda^{p-1-\delta}\left|B_{4 \varrho}-F(\lambda)\right| d \lambda .
\end{aligned}
$$

We write this as

$$
I_{0} \leq c\left[I_{1}+I_{2}\right]+c \delta\left[I_{3}+I_{4}\right] .
$$

To estimate $I_{i}, i=0,1, \ldots, 4$, we remark that by Lemma 2.4 ,

$$
\left|u(x)-u_{4_{\varrho}}\right| \leq c \varrho\left[M\left(|D u| \chi_{B_{4 \varrho}}\right)\right] \quad \text { for any } x \in B_{4 \varrho} ;
$$

therefore,

$$
|D \widetilde{u}| \leq|D u|+c\left[M\left(|D u| \chi_{B_{4 \ell}}\right)\right]
$$


To simplify the presentation we have collected the estimates of $I_{i}, i=$ $1,2,3,4$, in the Appendix at the end of the paper.

By those estimates, (3.6) becomes

$$
\begin{aligned}
I_{0} \leq & c\left(\eta^{1-\delta}+\delta^{1-\delta}+\frac{\delta}{1-\delta}\right) \int_{B_{4 \varrho}}|D u|^{p-\delta} d x \\
& +c\left(\eta^{1 /(1-p)}+\eta^{1-p}\right) \varrho^{n}\left(f_{B_{4}}|D u|^{t} d x\right)^{(p-\delta) / t} \\
& +c \delta^{-\delta} \int_{B_{2 \varrho}-B_{\varrho}}|D u|^{p-\delta} d x
\end{aligned}
$$

where $(p-\delta)_{*} \leq t<p-\delta, c=c(m, n, p, \alpha, \beta)$ and $\eta$ is a constant to be chosen at the end. Since $\widetilde{u}=u$ on $B_{\varrho}$, by (3.7) we see that at $x \in B_{\varrho / 2}$,

$$
\begin{aligned}
M(|D \widetilde{u}|) & \leq M\left(|D u| \chi_{B_{\varrho}}\right)+c f_{B_{4 \varrho}}|D \widetilde{u}| d x \\
& \leq M\left(|D u| \chi_{B_{\varrho}}\right)+c f_{B_{4 \varrho}} M\left(|D u| \chi_{B_{4 \varrho}}\right) d x .
\end{aligned}
$$

Let

$$
G=\left\{x \in B_{\varrho / 2}: M\left(|D u| \chi_{B_{\varrho}}\right)(x) \geq c f_{B_{4 \varrho}} M\left(|D u| \chi_{B_{4 \varrho}}\right) d y\right\} .
$$

Then $M(|D \widetilde{u}|) \leq c M\left(|D u| \chi_{B_{e}}\right)$ on $G$ and so, by Lemma 2.2 and Theorem 2.3 , if $0<2 \delta<p-1$ then

$$
\begin{aligned}
I_{0} & \geq c^{-1} \int_{B_{\varrho}} M(|D \widetilde{u}|)^{-\delta} M\left(|D u| \chi_{B_{\varrho}}\right)^{p} d x \\
& \geq c^{-1} \int_{G} M\left(|D u| \chi_{B_{\varrho}}\right)^{p-\delta} d x \\
& =c^{-1}\left[\int_{B_{\varrho / 2}} M\left(|D u| \chi_{B_{\varrho}}\right)^{p-\delta} d x-\int_{B_{\varrho / 2}-G} M\left(|D u| \chi_{B_{\varrho}}\right)^{p-\delta} d x\right] \\
& \geq c^{-1} \int_{B_{\varrho / 2}}|D u|^{p-\delta} d x-c \varrho^{n}(\underbrace{}_{B_{4_{\varrho}}} M\left(|D u| \chi_{B_{4 \varrho}}\right) d x)^{p-\delta} \\
& \geq c^{-1} \int_{B_{\varrho / 2}}|D u|^{p-\delta} d x-c \varrho^{n}\left({\underset{B}{B_{\varrho}}}_{\mid}|D u|^{t} d x\right)^{(p-\delta) / t}
\end{aligned}
$$

where $(p-\delta)_{*} \leq t<p-\delta$. 
From (3.8) and (3.9) we conclude that

$$
\begin{aligned}
\int_{B_{\varrho / 2}}|D u|^{p-\delta} d x \leq & c\left(\eta^{1-\delta}+\delta^{1-\delta}+\frac{\delta}{1-\delta}\right) \int_{B_{4 \varrho}}|D u|^{p-\delta} d x \\
& +c\left(\eta^{1-p}+\eta^{1 /(1-p)}\right) \varrho^{n}\left(\int_{B_{4 \varrho}}|D u|^{t} d x\right)^{(p-\delta) / t} \\
& +c \delta^{-\delta} \int_{B_{2 \varrho}-B_{\varrho / 2}}|D u|^{p-\delta} d x
\end{aligned}
$$

Now, we apply the "hole filling" method. Adding $c \delta^{-\delta} \int_{B_{\varrho / 2}}|D u|^{p-\delta} d x$ to both sides of (3.10) we get

$$
\begin{aligned}
\underset{B_{\varrho / 2}}{f}|D u|^{p-\delta} d x \leq & \frac{c}{c \delta^{-\delta}+1}\left(\eta^{1-\delta}+\delta^{1-\delta}+\delta^{-\delta}+\frac{\delta}{1-\delta}\right) \underset{B_{4 \varrho}}{ }|D u|^{p-\delta} d x \\
& +\frac{c}{c+1}\left(\eta^{1-p}+\eta^{1 /(1-p)}\right)\left(\underset{B_{4 \varrho}}{f}|D u|^{t} d x\right)^{(p-\delta) / t} .
\end{aligned}
$$

Notice that there exists $0<\delta_{1}<1$ such that if $0<\delta<\delta_{1}$ then

$$
\frac{c}{c \delta^{-\delta}+1}\left(\delta^{1-\delta}+\delta^{-\delta}+\frac{\delta}{1-\delta}\right)<\frac{c}{c+1 / 2} .
$$

Obviously $\delta_{1}$ depends on $c$ and therefore on $\alpha, \beta, p, m, n$.

If we choose $0<\eta<1$ such that

$$
\frac{c \eta}{c+1}<\vartheta \quad \text { where } \quad \frac{c}{c+1 / 2}<\vartheta<1,
$$

from the estimates above we have for $0<\delta<\delta_{1}$,

$$
f_{B_{\varrho} / 2}|D u|^{p-\delta} d x \leq \vartheta f_{B_{4 \varrho}}|D u|^{p-\delta} d x+\widehat{c}\left(f_{B_{4 \varrho}}|D u|^{t} d x\right)^{(p-\delta) / t}
$$

where $\widehat{c}$ depends on $\alpha, \beta, m, n, p$ but not on $\delta$. The result follows from Theorem 2.6 with an argument similar to the one of [GLS].

4. Appendix. We now proceed to the estimates of $I_{i}, i=1,2,3,4$.

Estimate of $I_{1}$. We have

$$
\begin{aligned}
I_{1} \leq & \int_{E\left(\lambda_{0}\right) \cap B_{2 \varrho}}|D \widetilde{u}|^{p} M(|D \widetilde{u}|)^{-\delta} d x \\
& +\int_{E\left(\lambda_{0}\right) \cap B_{2 \varrho}}|D u|^{p-1} M(|D \widetilde{u}|)^{1-\delta} d x .
\end{aligned}
$$


If $x \in E\left(\lambda_{0}\right)$, then $M(|D \widetilde{u}|) \leq \lambda_{0}$ and so at $x$ we find

$$
|D \widetilde{u}|^{p} M(|D \widetilde{u}|)^{-\delta} \leq M(|D \widetilde{u}|)^{p-\delta} \leq \lambda_{0}^{p-\delta} .
$$

Now suppose $0<\eta \leq 1 / 2$ and $|D u| \geq \eta^{-1} \lambda_{0}$. Then at $x \in E\left(\lambda_{0}\right)$,

$$
M(|D \widetilde{u}|) \leq \lambda_{0} \leq|D u| \eta
$$

and so

$$
|D u|^{p-1} M(|D \widetilde{u}|)^{1-\delta} \leq \eta^{1-\delta}|D u|^{p-\delta} .
$$

On the other hand, if $x \in E\left(\lambda_{0}\right)$ and $|D u|<\eta^{-1} \lambda_{0}$ we get

$$
|D u|^{p-1} M(|D \widetilde{u}|)^{1-\delta} \leq \eta^{1-p} \lambda_{0}^{p-\delta} .
$$

Then from (4.1)-(4.3), at $x \in E\left(\lambda_{0}\right) \cap B_{2 \varrho}$,

$$
\begin{aligned}
|D \widetilde{u}|^{p} M(|D \widetilde{u}|)^{-\delta}+|D u|^{p-1} M(|D \widetilde{u}|)^{1-\delta} & \\
& \leq c\left(\eta^{1-p} \lambda_{0}^{p-\delta}+\eta^{1-\delta}|D u|^{p-\delta}\right) .
\end{aligned}
$$
that

Using the definition of $\lambda_{0}$ given in (3.1) and relation (3.7), we remark

$$
\begin{aligned}
\eta^{1-p} \lambda_{0}^{p-\delta} & \leq c \eta^{1-p}\left(f_{B_{4 \varrho}} M\left(|D u| \chi_{B_{4 \varrho}}\right) d x\right)^{p-\delta} \\
& \leq c \eta^{1-p}\left(f_{B_{4 \varrho}} M\left(|D u| \chi_{B_{4 \varrho}}\right)^{t} d x\right)^{(p-\delta) / t}
\end{aligned}
$$

where $(p-\delta)_{*} \leq t<p-\delta$.

Finally, by (4.4) and the previuos remark, applying the Hardy-Littlewood theorem we get

$$
I_{1} \leq c \eta^{1-\delta} \int_{B_{4 \varrho}}|D u|^{p-\delta} d x+c \eta^{1-p} \varrho^{n}\left(f_{B_{4 \varrho}}|D u|^{t} d x\right)^{(p-\delta) / t}
$$

where $c=c(\beta, m, n, p)$.

Estimate of $I_{2}$. From the definition of $\widetilde{u}$,

$$
\begin{aligned}
I_{2} \leq & c \int_{B_{2 \varrho}-B_{\varrho}}|D \widetilde{u}| \cdot|D u|^{p-1} M(|D \widetilde{u}|)^{-\delta} d x \\
& +c \int_{B_{2 \varrho}-B_{\varrho}}|D \widetilde{u}|\left(\frac{\left|u-u_{\varrho}\right|}{\varrho}\right)^{p-1} M(|D \widetilde{u}|)^{-\delta} d x \\
& =c(I+I I) .
\end{aligned}
$$

Let $D_{1}$ be the set of all $x \in B_{2 \varrho}-B \varrho$ such that

$$
M(|D \widetilde{u}|)(x) \leq \delta M\left(|D u| \chi_{B_{4 \ell}}\right)(x)
$$


and set $D_{2}=\left(B_{2 \varrho}-B_{\varrho}\right)-D_{1}$. Then

$$
\begin{aligned}
I \leq & \int_{D_{1}}|D \widetilde{u}| \cdot|D u|^{p-1} M(|D \widetilde{u}|)^{-\delta} d x \\
& +\int_{D_{2}}|\varphi| \cdot|D u|^{p} M(|D \widetilde{u}|)^{-\delta} d x \\
& +\frac{c}{\varrho} \int_{D_{2}}\left|u-u_{4}\right| \cdot|D u|^{p-1} M(|D \widetilde{u}|)^{-\delta} d x .
\end{aligned}
$$

Next, from the definition of $D_{1}$ and the Hardy-Littlewood maximal theorem, we get

$$
\begin{aligned}
\int_{D_{1}}|D \widetilde{u}| \cdot|D u|^{p-1} M(|D \widetilde{u}|)^{-\delta} d x & \leq \int_{D_{1}} M(|D \widetilde{u}|)^{1-\delta}|D u|^{p-1} d x \\
& \leq c \delta^{1-\delta} \int_{B_{4}}|D u|^{p-\delta} d x .
\end{aligned}
$$

On the other hand, since $M\left(|D u| \chi_{B_{4 \varrho}}\right)(x) \geq\left(|D u| \chi_{B_{4 \varrho}}\right)(x)$, we get

$$
\int_{D_{2}}|\varphi| \cdot|D u|^{p} M(|D \widetilde{u}|)^{-\delta} d x \leq \delta^{-\delta} \int_{D_{2}}|D u|^{p-\delta} d x \leq \delta^{-\delta} \int_{B_{2}-B_{\varrho}}|D u|^{p-\delta} d x .
$$

Finally, by Young's inequality,

$$
\begin{aligned}
\int_{D_{2}} \frac{\left|u-u_{4 \varrho}\right|}{\varrho}|D u|^{p-1} M(|D \widetilde{u}|)^{-\delta} d x \\
\quad \leq \delta^{-\delta} \int_{D_{2}} \frac{\left|u-u_{4 \varrho}\right|}{\varrho}|D u|^{p-1-\delta} d x \\
\leq \delta^{-\delta}\left(\int_{D_{2}}|D u|^{p-\delta} d x+\int_{B_{4 \varrho}}\left(\frac{\left|u-u_{4 \varrho}\right|}{\varrho}\right)^{p-\delta} d x\right) \\
\leq \delta^{-\delta} \int_{B_{2 \varrho}-B_{\varrho}}|D u|^{p-\delta} d x+c \varrho^{n}\left({\underset{B}{B_{\varrho}}}_{\mid}|D u|^{t} d x\right)^{(p-\delta) / t}
\end{aligned}
$$

where $(p-\delta)_{*} \leq t<p-\delta$.

By the above estimates we can conclude that

$$
\begin{aligned}
I \leq & c \delta^{1-\delta} \int_{B_{4 \varrho}}|D u|^{p-\delta} d x+c \delta^{-\delta} \int_{B_{2 \varrho}-B_{\varrho}}|D u|^{p-\delta} d x \\
& +c \varrho^{n}\left(\int_{B_{4 \varrho}}|D u|^{t} d x\right)^{(p-\delta) / t} .
\end{aligned}
$$


To estimate $I I$ we remark that

$$
I I \leq \int_{B_{2 \varrho}-B_{\varrho}} M(|D \widetilde{u}|)^{1-\delta}\left(\frac{\left|u-u_{4 \varrho}\right|}{\varrho}\right)^{p-1} d x .
$$

Then by relation (3.7) and Young's inequality we get

$$
\begin{aligned}
I I \leq & \eta^{1-\delta} \int_{B_{2 \varrho}-B_{\varrho}} M(|D \widetilde{u}|)^{p-\delta} d x \\
& +c \eta^{-(1-\delta)^{2} /(p-1)} \int_{B_{2 \varrho}-B_{\varrho}} M\left(\frac{\left|u-u_{4 \varrho}\right|}{\varrho}\right)^{p-\delta} d x \\
\leq & c \eta^{1-\delta} \int_{B_{2 \varrho}-B_{\varrho}}\left[M^{2}\left(|D u| \chi_{B_{4}}\right)\right]^{p-\delta} d x \\
& +c \eta^{-(1-\delta)^{2} /(p-1)} \varrho^{n}\left(\int_{B_{4 \varrho}}|D u|^{t} d x\right)^{(p-\delta) / t}
\end{aligned}
$$

where $0<\eta<1 / 2$ and $(p-\delta)_{*} \leq t<p-\delta$.

Finally, from the Hardy-Littlewood maximal theorem we deduce that

$$
I I \leq c \eta^{1-\delta} \int_{B_{4} \varrho}|D u|^{p-\delta} d x+c \varrho^{n} \eta^{1 /(1-p)}\left(\int_{B_{4 \varrho}}|D u|^{t} d x\right)^{(p-\delta) / t} .
$$

Then from (4.5)-(4.7),

$$
\begin{aligned}
I_{2} \leq & c\left(\delta^{1-\delta}+\eta^{1-\delta}\right) \int_{B_{4 \varrho}}|D u|^{p-\delta} d x+c \varrho^{n} \eta^{1 /(1-p)} \\
& \times\left(\int_{B_{4 \varrho}}|D u|^{t} d x\right)^{(p-\delta) / t}+c \delta^{-\delta} \int_{B_{2 \varrho}-B_{\varrho / 2}}|D u|^{p-\delta} d x .
\end{aligned}
$$

Estimate of $I_{3}$ and $I_{4}$. By using (3.7) and the Hardy-Littlewood maximal theorem, we get

$$
\begin{aligned}
I_{3} & =\int_{B_{4}}|D u|^{p-1} d x \int_{\lambda_{0}}^{M(|D \widetilde{u}|)} \lambda^{-\delta} d \lambda \\
& \leq \frac{1}{1-\delta} \int_{B_{4}}|D u|^{p-1} M(|D \widetilde{u}|)^{1-\delta} d x \\
& \leq \frac{c}{1-\delta} \int_{B_{4}}|D u|^{p-\delta} d x .
\end{aligned}
$$


On the other hand, also by (3.7),

$$
\begin{aligned}
I_{4} & =\frac{c}{p-\delta} \int_{B_{4 \varrho}}|D \widetilde{u}|^{p-\delta} d x \leq \frac{c}{p-\delta} \int_{B_{4 \varrho}}|D u|^{p-\delta} d x \\
& \leq \frac{c}{1-\delta} \int_{B_{4 \varrho}}|D u|^{p-\delta} d x .
\end{aligned}
$$

\section{References}

[AF] E. Acerbi and N. Fusco, Semicontinuity problems in the calculus of variations, Arch. Rational Mech. Anal. 86 (1984), 125-145.

[Do] A. Dolcini, A uniqueness result for very weak solutions of p-harmonic type equations, Boll. Un. Mat. Ital. A (7) 10 (1996), 71-84.

[G] F. Gehring, $L^{p}$ integrability of the partial derivatives of a quasiconformal mapping, Acta Math. 130 (1973), 265-277.

[GLS] D. Giachetti, F. Leonetti and R. Schianchi, On the regularity of very weak minima, Proc. Roy. Soc. Edinburgh Sect. A 126 (1996), 287-296.

[Gi] M. Giaquinta, Multiple Integrals in the Calculus of Variations and Nonlinear Elliptic Systems, Ann. of Math. Stud. 105, Princeton Univ. Press, 1983.

[GG] M. Giaquinta and E. Giusti, On the regularity of the minima of variational integrals, Acta Math. 148 (1982), 31-46.

[Gu] E. Giusti, Metodi diretti nel Calcolo delle Variazioni, Un. Mat. Ital., 1984.

[GIS] L. Greco, T. Iwaniec and C. Sbordone, Variational integrals of nearly linear growth, preprint, Dip. di Mat. e Appl. R. Caccioppoli, Napoli.

[Iw] T. Iwaniec, p-Harmonic tensors and quasiregular mappings, Ann. of Math. 136 (1992), 589-624.

[IS] T. Iwaniec and C. Sbordone, Weak minima of variational integrals, J. Reine Angew. Math. 454 (1994), 143-161.

[Le] J. Lewis, On very weak solutions of certain elliptic systems, Comm. Partial Differential Equations 18 (1993), 1515-1537.

[Mo] G. Moscariello, Weak minima and quasiminima of variational integrals, Boll. Un. Mat. Ital. B 11 (1997), 355-364.

$[\mathrm{Mu}]$ B. Muckenhoupt, Weighted norm inequalities for the Hardy maximal function, Trans. Amer. Math. Soc. 165 (1972), 207-226.

[Sb] C. Sbordone, Quasiminima of degenerate functionals with non polynomial growth, Rend. Sem. Mat. Fis. Milano 59 (1989), 173-184.

[To] A. Torchinsky, Real-Variable Methods in Harmonic Analysis, Pure and Appl. Math. 123, Academic Press, 1986.

D.I.I.M.A.

Università degli Studi di Salerno

Via S. Allende

84081 Baronissi (SA), Italy

E-mail: moscar@matna2.dma.unina.it 